\title{
A percepção do cuidador familiar sobre a recuperação física do idoso em condição de incapacidade funcional
}

\author{
The family caretaker perception \\ about the elderly physical recovery with functional disability
}

Nilda Emiko N ozaki Israel ${ }^{1}$

O séias Guimarães deAndrade ${ }^{2}$ (in memoriam)

Jorge Juarez Vieira Teixeira ${ }^{3}$

${ }^{1}$ Hospital Universitário Regional de M aringá, Universidade Estadual de M aringá. Av. M andacaru 1.590, Vila Vardelina. 87080-000 Maringá PR. nildaisrael@yahoo.com.br

${ }^{2}$ Departamento de

Enfermagem, Centro de

Ciências da Saúde,

Universidade Estadual de

M aringá.

${ }^{3}$ Departamento de Análises

Clínicas, Centro de

Ciências da Saúde,

Universidade Estadual de

M aringá.
Abstract The objective of this study was to identify perceptions of family caretakers about the process of physical rehabilitation of elderly people were discharged from hospital on condition of physical dependence. This is a qualitative study, conducted from July to August 2005 in which six family caretakers were interviewed in their own homes, a month after hospital discharge of the elderly. By means of transcription and thematic analysis five themes emerged, highlighting: popular concept of rehabilitation, the process of functional disability, the main resources used and the motivation of the elderly and the caretaker. We found that these family caretakers perceive the physical recovery in a combined way with the care, since their actions are held for the full re covery of the elderly.

Key words Elder, Family, Activities of daily living, Rehabilitation, Caretaker
Resumo 0 objetivo deste estudo foi o de identificar percepções de cuidadores familiares sobre 0 processo de recuperação física de idosos que receberam alta hospitalar em condição de dependência física. Trata-se de um estudo qualitativo, realizado no período de julho a agosto de 2005, em que seis cuidadores familiares foram entrevistados, em seu próprio domicílio, um mês apósa alta hospitalar do idoso. M ediantea transcrição eanálise temática, surgiram cincos temas, em destaque: conceito popular de reabilitação, o processo da incapacidade funcional, principais recursos utilizados e a motivação do idoso e do cuidador. 0 bservou-se que estes cuidadores familiares percebem a recuperação física de maneira unificada com o cuidado, uma vez que as suas ações são realizadas para a recuperação integral do idoso. Palavras-chave Idoso, Família, Atividades cotidianas, Reabilitação, Cuidador 


\section{Introdução}

A alta frequência de doenças crônicas ea longevidade da população brasileira são apontadas como as principais causas de aumento de idosos com incapacidade funcional ${ }^{1,2}$. Por outro lado, há evidências de redução de declínio funcional da população idosa, indicando um envelhecimento mais saudável ${ }^{1}$.

A incapacidade é a restrição, resultante de uma deficiência, da habilidade para desempenhar uma atividade considerada normal para o ser humano. Surge como consequência direta ou éa resposta do indivíduo a uma deficiência psicológica, física, sensorial ou outra e reflete os distúrbios da própria pessoa, nas atividades e comportamentos essenciais à vida diária ${ }^{3}$.

A principal ideia que norteia as questões de saúde do idoso diz respeito à capacidade que ele tem de manter uma vida autônoma e independente, expressa pela capacidade de autodeterminação e execução de atividades de vida diária, sem necessidade de ajuda durante a velhicet.

De acordo com a Organização Mundial da Saúde ${ }^{5}$, a incapacidade funcional é uma dificuldade, devido a uma deficiência, para realizar atividades típicas e pessoalmente desejadas na sociedade. Pode levar o idoso à condição de dependência, à necessidade de auxílio para realizar alguma atividade de vida diária ${ }^{6}$. Características como 0 analfabetismo, a idade, saúde mental, algumas doenças como o AVC (acidente vascular cerebral) e história de internação hospitalar estão associadas com dependência moderada/ grave, que sugerem uma rede causal complexa para o declínio da capacidade funcional ${ }^{7}$.

A hospitalização do idoso pode trazer como repercussão uma diminuição da capacidadefuncional, muitas vezes, irreversível. Eleé mais susceptível devido ao repouso prolongado no leito, associado aos fatores fisiológicos e doenças incapacitantes queacometem com maisfrequência esta faixa etária ${ }^{7}$. Esta situação pode ser ainda agravada pelo fato de que os cuidados hospitalares frequentementeestão focados no tratamento de doenças agudas e a função física ea cognitiva, que são fatores que mais afetam o prognóstico de independência do idoso, geralmente são deixadas para segundo plano $0^{8}$.

A perda da capacidade funcional, especialmente na sua dimensão física, está associada à predição de fragilidade, dependência, institucionalização, gerando altos custos para a família e sistema de saúde ${ }^{2,9}$. N este sentido, a recuperação da função física está relacionada a uma melhor qualidade de vida, tornando-se item fundamental na saúde do idoso8,10.

Dentre as várias questões que envolvem a capacidade funcional, como por exemplo, a redução de custo, em muitos países e no Brasil, a tendência é indicar a permanência dos idosos incapacitados em suas casas sob os cuidados de sua família, tornando-se o cuidador a principal ferramenta na recuperação e cuidado do idoso com incapacidade funcional ${ }^{2,11}$.

A disponibilidade de uma esposa ou parente, independentemente da qualidade ou quantidade de suporte que eles oferecem, tem sido associada ao aumento da mobilidade e consequente meIhora da função ${ }^{12}$.

Cuidadores relatam que raramente recebem informações claras a respeito da doença, orientação ou apoio para os cuidados, nem indicação de um serviço para prosseguir o tratamento ${ }^{13}$. Embora a educação de pacientes e familiares seja reconhecida como importante componente de uma boa prática na reabilitação, relativamente pouco se tem estudado sobre as suas necessidades de aprendizado. Portanto, compreender as dúvidas e interesses que os cuidadores possuem pode garantir que a informação dada seja relevante ${ }^{14}$.

A presentepesquisa teve como objetivo identificar características e percepções do cuidador familiar quanto ao processo de recuperação física de idosos que receberam alta hospitalar em condição de incapacidade funcional.

\section{M etodologia}

Pesquisa qualitativa e descritiva, realizada no período de 21 dejulho a 21 deagosto, no município de M aringá (PR), com seis cuidadores de idosos que receberam alta de dois hospitais públicos em condição de incapacidade funcional.

$\mathrm{O}$ projeto de pesquisa foi aprovado pelo $\mathrm{Co}$ mitêPermanente deÉtica em Pesquisa Envolvendo Seres Humanos (COPEP), da Fundação Universidade Estadual de M aringá.

Para avaliar a capacidade funcional dos idosos internados, foi utilizado o índice de Barthel, que avalia as atividades básicas de vida diária (ABVD). Elas correspondem aos níveis mais graves de deficiência das aptidões físicas, relevantes aos pacientes frágeis e ou institucionalizado $\mathrm{s}^{15}$. A pontuação varia de zero (máxima dependência) a cem (independente).

Os critérios de inclusão dos idosos foram possuir sessenta anos ou mais, ter sido internado no Hospital Universitário ou Municipal de 
M aringá e ter tido alta hospitalar em condição de incapacidade funcional. $E$ do cuidador foram ter algum grau de parentesco com o idoso, residir na cidade de $M$ aringá e concordar em participar do estudo.

No primeiro momento, foi realizada a avaliação do idoso, na alta hospitalar eum mês após, no domicílio. No segundo momento, foram identificados e contatados os seus respectivos cuidadores.

Em um segundo momento, foi utilizada entrevista semi-estruturada com os cuidadores, para servir deorientação eguia, permitindo também flexibilidade para temas e questões de rele vância para a pesquisa ${ }^{16}$. A entrevista foi realizada no domicílio pela própria pesquisadora, me diante assinatura do termo de consentimento livre eEsclarecido (TCLE), e gravadas para posterior transcrição e melhor compreensão dos dados obtidos.

A pós leitura do material, os dados foram organizados, seu conteúdo analisado através da análise temática, que consiste em desvelar os núcleos de sentido de um discurso, podendo ser apresentadas através de uma palavra, frase ou resumo ${ }^{17}$. Os principais temas desenvolvidos em relação aos cuidadores foram o conceito popular de reabilitação, o processo de incapacidade funcional e os recursos utilizados pela família para promover a recuperação, o conhecimento sobre a doença e sua preven ção, a motivação do idoso no processo de recuperação e o cuidador.

A análise dos temas foi de natureza descritiva, com suporte da literatura científica.

\section{Resultados ediscussão}

Os idosos utilizam os serviços hospitalares de maneira mais intensiva que os demais grupos etários, envolvendo maiores custos, implicando um tratamento de duração mais prolongada e de recuperação mais lenta e complicada ${ }^{18}$.

A Tabela 1 destaca os idosos participantes do estudo que apresentaram incapacidade funcional na alta hospitalar, a maioria com idade acima dos setenta anos, tendo no diagnóstico de internação a presença de AVC, o qual representa uma das principais causas de mortee morbidez no mundo ocidental ${ }^{11}$. Por motivo de enfermidade e hospitalização, a maioria apresentou baixo índice de Barthel, indicando aumento da depen dênciafuncional, sendo que após a alta hospitalar três idosos apresentaram aumento do índice, caracterizando melhora da função, resultado semel hante encontrado no estudo deKawasaki eDiogo ${ }^{19}$, que avaliou independência funcional no momento, durantea internação, na alta hospital ar eum mês após o retorno ao domicílio. Este estudo apontou piora dos escores na alta hospitalar e melhora das tarefas de autocuidado, do controle deurina, de transferências e autocuidado após retorno ao domicílio. Este mesmo autor evidencia que a própria instituição hospitalar pode potencializar os declínios funcionais por falta de adaptação ambiental como, por exemplo, as barras deapoio. Por outro lado, Siqueira et al. ${ }^{4}$ avaliaram somente durante o período de internação, na entrada ena alta, e a maioria dos idosos não sofreu alterações funcionais, talvez porque a pesquisa foi realizada em uma enfermariageriátrico-gerontológica enão clínica médica. Outro estudo acrescenta o fato de que idosos com piora do nível de independência funcional durante a hospitalização tiveram mais chances de óbito dentro de três meses após a alta hospitalar ${ }^{20}$.

A visibilidadesocial do cuidador émuito restrita em países em que o envelhecimento da população vem acontecendo há poucas décadas,

Tabela 1. Características dos idosos de duas instituições hospitalares públicas em condição de incapacidade funcional. Maringá (PR), julho a agosto de 2004.

\begin{tabular}{lcclcc}
\hline Idoso (I) & I dade & Gênero & Diagnóstico de internação & Barthel no hospital & Barthel no domicílio \\
\hline $\mathrm{I}_{1}$ & 80 & M & ICC+HAS+AVC & 25 & 15 \\
$\mathrm{I}_{2}$ & 75 & $\mathrm{~F}$ & AVC+HAS & 40 & 80 \\
$\mathrm{I}_{3}$ & 85 & $\mathrm{~F}$ & ITU+BCP+DM +AVC & 0 & 0 \\
$\mathrm{I}_{4}$ & 74 & $\mathrm{~F}$ & Úlceras+AIzheimer & 0 & 0 \\
$\mathrm{I}_{5}$ & 65 & M & AVC+Chagas & 10 & 40 \\
$\mathrm{I}_{6}$ & 78 & M & Pn+AVC+IAM & 30 & 75 \\
\hline
\end{tabular}

Legendas: ICC = insuficiência cardíaca congestiva; HAS = hipertensão arterial sistêmica; $\mathrm{AVC}$ = acidente vascular cerebral; ITU = infecção do trato urinário; $\mathrm{BCP}=$ broncopneumonia; $\mathrm{DM}$ = diabetes mellitus; $\mathrm{PN}=$ pneumonia; $\mathrm{IAM}=$ infarto agudo do miocárdio. 
como o Brasil. Geralmente estão presentes na designação da pessoa que, preferencialmente, assume os cuidados pessoais do idoso incapacitado: parentesco (cônjuges); gênero (feminino); proximidade física (vive junto) e proximidade afetiva (conjuga, pais efilhos) ${ }^{2}$. As principais características dos cuidadores desta pesquisa podem ser observadas na Tabela 2. Todas as participantes pertenciam ao gênero feminino, faixa etária de 22 a 77 anos e o parentesco em relação ao idoso, filha, esposa eneta. 0 tempo de estudo destes cuidadores variou de um a onze anos. $D$ as seis cuidadoras, cinco relataram comprometimento da saúde física, prevalecendo a expressão dor em quatro. A mai oria das cuidadoras relataram oferecer algum tipo de assistência nas ABVD do idoso há mais de três anos. Este perfil vai ao encontro de outros estudos realizados com cuidadores de idosos dependentes ${ }^{21,22}$.

De acordo com o discurso dos cuidadores entrevistados, emergiram cinco temas principais, apresentados a seguir.

Um conceito popular de reabilitação

(Tema 1)

A intervenção precoce de reabilitação após a presença de um AVC pode melhorar o processo de recuperação e minimizar a incapacidade funcional e, consequentemente, contribuir para aumento da satisfação do paciente e redução dos custos de cuidado a longo prazo ${ }^{11}$.

De acordo com o cuidador 3(C3) e 4(C4), a recuperação física éreabilitar eestá intimamente relacionada à melhora dos movimentos eà realização das ABVD, é não depender de ninguém para viver: "É reabilitar, é ela voltar a andar, a falar, comer com as próprias mãos, é ela voltar novamente a se movimenta". (C4)
"É reabilitar novamentepara uma vida digna, né, uma vida justa, é poder fazer pelos menos as coisinhas dela. Não étrabalhar, mas pelo menos se virar com o próprio corpo dela". (C3)

A partir deste tema, pode-se definir um conceito popular de reabilitação, como sendo o retorno para uma vida normal e real ização das atividades essenciais sem a ajuda de outras pessoas. Este conceito vai ao encontro do objetivo mais importante na reabilitação do idoso, que é o retorno das condições de vida anteriores à doença ${ }^{23}$. Embora muitas vezes a recuperação não seja total, principalmentenos casos de pacientes com AVC, a família e o paciente devem ser orientados sobre a possi bilidade do idoso não conseguir recuperar total mente a capacidade funcional anterior ao AVC.

0 processo de incapacidade funcional e os recursos utilizados pela família para promover a recuperação (Tema 2)

Não somente as enfermidades que afetam 0 aparelho locomotor, mas também enfermidades sistêmicas muito prevalentes, como a insuficiência cardíaca, a pneumonia, a demência, semanifestam com perda da função do idoso ${ }^{15}$. O AVC éuma das principais doenças que mais causam incapacidade no adulto e o grau de incapacidade vai determinar o nível de dependência por assistência ${ }^{23}$.

Fatores psicológicos, educação, culturae condições de comorbidade também aumentam as chances do idoso apresentar incapacidade ${ }^{24}$.

Para os cuidadores, além das doenças, as causas da perda da função estiveram associadas à idade, inatividade ou eventos importantes, como a perda de um ente querido e aposentadoria:

"Parou de trabalhar e depois ele se acomodou, filha, ficou assim sem fazer nada. Ele pegou erisi-

Tabela 2. Características dos cuidadores familiares. M aringá (PR), setembro a novembro de 2004.

\begin{tabular}{lcclccc}
\hline Cuidador (C) & Idade & Gênero & Parentesco & Tempo de estudo (anos) & Tempo de cuidado & Problemas de saúde \\
\hline $\mathrm{C}_{1}$ & 77 & $\mathrm{~F}$ & Esposa & 0 & 3 anos & $\begin{array}{l}\text { Diabetes, pressão alta, } \\
\text { dor no corpo }\end{array}$ \\
$\mathrm{C}_{2}$ & 52 & $\mathrm{~F}$ & Filha & 4 & 4 anos & $\begin{array}{l}\text { Dor no braço } \\
\mathrm{C}_{3}\end{array}$ \\
50 & $\mathrm{~F}$ & Filha & 8 & 8 meses & $\begin{array}{l}\text { Ciático, hérnia de hiato, } \\
\text { reumatismo no sangue }\end{array}$ \\
$\mathrm{C}_{4}$ & 22 & $\mathrm{~F}$ & N eta & 11 & 4 anos & Dor no cotovelo \\
$\mathrm{C}_{5}$ & 64 & $\mathrm{~F}$ & Esposa & 3 & 1 mês & Dor nas costas \\
$\mathrm{C}_{6}$ & 51 & $\mathrm{~F}$ & Filha & 2 & 4 anos & - \\
\hline
\end{tabular}


pela, atrofiou as pernas deleeagora também já deu outros problemas no coração, na pressão alta, essas coisas, deu derramenele também, né, ele ficou com uma perna esquecida. (C1)

A perda daindependência eautonomiaacontecee aumenta aos poucos e parecelimitar a qualidade de vida do idoso e, consequentemente, pode aumentar a sobrecarga de seu cuidador, refletida no discurso do próprio cuidador e pelo relato da presença de dores osteomusculares (Tabela 2): "Eleusava a bengala dentro decasa, ele seajudava um pouco. Detrêsanos pra cá, étudo eu que faço". (C1)

Para a recuperação física do idoso, o fisioterapeuta foi reconhecido pelos cuidadores como o principal profissional responsável. No entanto, dificuldades no meio de transporte comprometem o seguimento do tratamento disponível na rede pública de saúde, denotando a necessidade da disponibilização dos profissionais de saúde no domicílio ou transporte adequado para algum centro de reabilitação: "Se ele tivesse uma fisioterapia toda semana, acho eu, acho que ele se recuperava, mas é muito difícil pra levar, carro e condições pra levar, a gente nunca arrumou". (C6)

Elas também referiram que o fisioterapeuta solicita alguns cuidados na alta hospitalar e, por isso, muitas vezes é necessário um preparo para a alta hospitalar por partedesteprofissional, pois não é possível absorver as instruções dadas duranteo período de internação para o cuidado do idoso no domicílio: "Eu queria saber como fazer fisioterapia, né, é que a gente não sabe mexer com essas coisas, né, difícil, ena hora do dia, você, cabe ça ruim, né, e você nem consegue aprender também, né, não consegue aprender nada assim ligeiro, né, imediatamente". (C5)

Às vezes, estas recomendações do fisioterapeuta podem ser consideradas como uma sobrecarga, pois aumentam as tarefas que os cuidadores têm a desempenhar; mas, apesar das dificuldades, as cuidadoras realizam al guns cuidados de fisioterapia ao idoso, aprendidos através da observação durante a internação e também intuitivamente, tais como o estímulo para realizar as ABVD, mobilização passiva, transferências e promoção de analgesia através de pomadas, massagens e compressas mornas: "M exo com os pés, mexo com as mãos, pegar o braço dela que tá inchado, levantar assim umas quinze ou vintevezes. Eu faço assim, do pé, eu não sei, mas eu só faço assim do pé". (C4)

Os cuidadores têm adquirido equipamentos parafacilitar a assistência ao idoso principalmente nas atividades que exigem maior esforço, como o banho e mobilização para dentro e fora da residência. Os equipamentos mais adquiridos foram cadeira de banho, cadeira de rodas, esfignomanômetro, estetoscópio, termômetro e inalador. Por saber da importância do controle da pressão arterial, algumas cuidadoras providenciaram aprender manusear e comprar o esfignomanômetro e o estetoscópio.

A pesar de não serem orientadas para tal, a própria família realizou adaptações ambientais no domicílio para idosos, com o objetivo de facilitar a segurança emovimentação do próprio idoso no domicílio, como rampas, apoio no banheiro, colocação de piso menos escorregadio. Pensando na segurança do idoso, os cuidadores também trocaram os utensílios de vidro por plástico.

Sobre o conhecimento da doença e sua prevenção (Tema 3)

O conhecimento sobrea doença e incapacidade por parte do cuidador pode diminuir a tensão na prestação de cuidados e facilitar a prevenção secundária ereabilitação do idoso ${ }^{14}$. Todososidosos da pesquisa tinham história de AVC e, no entanto, os seus cuidadores demonstraram possuir pouco ou nenhum conhecimento sobreesta principal doença incapacitante, o que denota falta de capacitação do cuidador e ausência de políticas públicas frente a esta problemática: “Ele teve derramee ficou assim, só que não sei te dizer não o que que é isso, não. Eu acho que é um coágulo no cére bro, não é? Eu imagino que é, não sei". (C3)

É importante, também, o conhecimento do potencial elimitação funcional do idoso, dos tratamentos necessários, dos medicamentos prescritos e recursos disponíveis, pois isto pode garantir à família informações adequadas e relevantes às demandas de responsabilidadena prestação de atendimento ao idoso dependente, uma vez que o cuidador familiar e a família formam uma unidade de cuidado e são a principal fonte de suporte social e econômico ${ }^{14,25}$.

\section{A motivação do idoso \\ no processo de recuperação (Tema 4)}

Os idosos frequentemente estão ansiosos devido à incapacidade física, dor e alterações no estilo de vida, o que pode resultar muitas vezes em depressão $0^{25}$. 0 humor deprimido, sentimentos de tristeza, desesperança, além da perda de interesse em realizar as atividades previamente prazerosas, são fatores que dificultam o cuidado e a recuperação do idoso como relatado pelos 
cuidadores: "A fisioterapia ali no posto, no hospital, ela não deixa fazer, tenta fazer os movimentos com os braços, ela fica nervosa, endurece os braços enão deixa fazer. E obrigar ela é pior, porque disse que ela não é pra passar nervoso, então tem que deixar os exercícios". (C3)

"A gentetenta fazer alguma coisa, levar ele pra sair de casa, só que ele não quer mesmo, ele não quer fazer nada". (C6)

0 que demonstra a íntima relação entre a função física eemocional na recuperação do idoso, a complexidade da problemática e necessidade de uma abordagem global, multidimensional e interdisciplinar, como preconizado na Política Nacional de Saúde da Pessoa Idosa ${ }^{26}$.

\section{O cuidador (Tema 5)}

As cuidadoras relataram desgaste físico e emocional decorrentes da tarefa de cuidar de um idoso. Sua saúde está comprometida, pois além de enfermidades sistêmicas como, por exemplo, a hipertensão arterial, elas queixaram-se de problemas osteomusculares surgidos devido ao manejo do idoso: "Eu fiquei com dor no braço direito, ainda tá doendo até hoje". (C2)

Devido ao envolvimento com o cuidado do idoso, da casa e da família, muitos cuidadores deixam de cuidar de si mesmos, recorrendo à automedicação para solucionar os seus problemas de saúde: "Uma vez por ano eu arrumo minha unha earrumo meu cabel o devez em quando". (C1)

"O que alivia minhas dores éum remédio que minha amiga traz do Paraguai". (C3)

São várias tar efas envolvidas no dia a dia do cuidador, principalmente a medicação, a higiene e alimentação do idoso. Além destas tarefas, elas realizam tarefas domésticas da própria casa. 0 apoio é recebido por membros da família nas atividades que requerem maior esforço, como 0 banho e transferências, e quando podem também ajudam na esfera econômica.

Como sendo o principal provedor de cuidado ao idoso, muitas vezes elas são privadas do convívio social e, mesmo nas raras vezes queconseguem sair, não conseguem aproveitar o momento por estarem constantemente preocupadas com o bem-estar do idoso em casa: "Eu perdi praticamente um pouco da minha vida, que era pra mim tá fazendo vestibular, faculdade". (C4)

Existe uma relação entre o estresse decorrente dos papéis que as mulheres exerciam (cuidadoras, mãe, esposa) sobre a expectativa otimista: a presença de estresse durante mais de um ano decresceu o otimismo ${ }^{27}$. Provavelmente, por isso, a expectativa otimista de recuperação foi encontrada somente em uma cuidadora. Esta estava cuidando de sua mãe há apenas um mês: "Tá recuperando rápido, tenho certeza que vai ficar bem". (C2).

Outras relataram uma expectativa indiferente ou negativa em relação à recuperação do idoso: "Acabou da gentenão ter mais direito a comer com as próprias mão, acho quea vida da gente começa a terminar, acho que não tem mais graça". (C3)

\section{Considerações finais}

Os cuidadores familiares não percebem o processo de recuperação física de forma dicotomizada do cuidado realizado e suas ações estão voltadas para a recuperação ou melhora de forma integral de seus idosos.

A recuperação física parece ser o mesmo que recuperar a independência das ABVD. É ser independente e não necessitar de outra pessoa para o próprio cuidado. 0 acesso ao tratamento de re abilitação, como a fisioterapia, é muitas vezes penoso, uma vez que envolve a locomoção do idoso de sua residência para outro local, como clínica ou outro centro de saúde.

As dificuldades para cuidar do idoso são aumentadas devido a outras atividades que elas desempenham, como esposa, mãe e até avó, o que contribui para um comprometimento da qualidade de assistência, qualidade de vida do próprio cuidador e, portanto, uma expectativa pessimista em relação à recuperação física do idoso.

Sendo assim, torna-sefácil estabelecer um círculo vicioso: doença, incapacidadefuncional, falta de tratamento para reabilitação, agravamento da doença e, consequentemente, aumento da carga para o sistema público de saúde e para a família. Lembrando também que esteciclo também pode ser repetido com o cuidador, uma vez que el efica suscetível a adoecer.

Como o cuidar é um processo interativo entre o cuidador e o ser cuidado, o que afeta o cuidador, invariavelmente afeta também o idoso; por isso, medidas de apoio, como 0 atendimento domiciliar de serviços de saúde e melhora na capacidade funcional do idoso, podem ser tomadas com o objetivo de melhorar a qualidade de vida de ambos.

As limitações deste estudo se referem ao pequeno tamanho da amostra, pacientes oriundos exclusivamente do serviço público e não pertencentes a uma amostra probabi lística. Este estudo pode ser utilizado como exemplo em outras re 
giões do país, com amostras maiores tanto de idosos quanto de cuidadores do serviço público e do privado.

Pesquisas desta natureza podem ser utilizadas como uma ferramenta importante no processo de educação em saúde, pois é possível a percepção das necessidades do usuário e do seu cuidador.

\section{Colaboradores}

NEN I srael trabalhou na concepção, delineamento, interpretação dos dados e redação do artigo; OG Andrade trabalhou na metodologia e interpretação dos dados e JJV Teixeira trabalhou na revisão crítica e redação final.

\section{Referências}

1. Parahyba MI, Simões CCS. A prevalência de incapacidade funcional em idosos no Brasil. Cien Saude Colet 2006; 11(4):967-974.

2. Karsch UM . Idosos dependentes: famílias e cuidadores. Cad Saude Publica 2003; 19(3):861-866.

3. Amiralian MLT, Pinto EB, Ghirardi MIG, Lichiting I, Masini EFS, Pasqualin L. Conceituando deficiência. Rev. Saude Publica 2000; 34(1):97-103.

4. Siqueira $A B$, Cordeiro RC, Perracini MR, Ramos LR. Impacto funcional da internação hospitalar de pacientes idosos. Rev. Saude Publica 2004; 38(5):687694.

5. World Health Organization. Disability prevention and rehabilitation. Geneva: WH O; 1981.

6. Machado CJ, Perpétuo IH O, Camargos MCS. Expectativa de vida com incapacidade funcional em idosos em São Paulo, Brasil. Rev. Panam. Salud Públ. I Pan Am. J. Public Health 2005; 17(5-6):379-386.

7. Rosa TMC, Benício MHD, Latorre M RDO, Ramos LR. Fatores determinantes da capacidade funcional entre idosos. Rev. Saude Publica 2003; 37(1):40-48.

8. Graf C. Functional decline in hospitalized older adults. AJN 2006; 106(1):58-67.

9. Chaimovicz F. A saúde dos brasileiros às vésperas do século XXI: problemas, projeções e alternativas. Rev. Saude Publica 1997; 31:184-200.

10. OPAS. OMS. 36ạ Sessión Del subcomité de planificación y programación del comité ejecutivo. La salud y el envejecimento. Washington, D.C.: OPAS/OM S; 2002.

11. Duncan PW, Bates RZB, Choi JY, Glasberg JJ, Graham GD, Katz RC, Lamberty K, Reker D. Management of adult stroke rehabilitation care: a clinical practice guideline. Stroke 2005; 36:e100-e143.

12. Palmer S, Glass TA. Family function and stroke recovery: a review. Rehabil Psychol 2003; 48(4):255265. 
13. Caldas CP. Envelhecimento com dependência: responsabilidades e demandas da família. Cad Saude Publica 2003; 19(3):773-781.

14. M CLennan M, Anderson GS, Pain K. Rehabilitation learning needs: patient and family perceptions. Patient Educ Couns 1996; 27:191-199.

15. Paixão Jr. CM, Reichenheim ME. Uma revisão sobre instrumentos de avaliação do estado funcional do idoso. Cad Saude Publica 2005; 21(1):7-19.

16. M inayo MCS. 0 desafio do conhecimento: pesquisa qualitativa em saúde. 5a ed. São Paulo: Hucitec; Rio de Janeiro: Abrasco; 1998.

17. Bardin L. Análise de conteúdo. Lisboa: Edições 70; 1994.

18. Coelho Filho JM. Modelos de serviços hospitalares para casos agudos em idosos. Rev Saúde Pública 200; 34:666-671.

19. Kawasaki K, Diogo MJDE. Impacto da hospitalização na independência funcional do idoso em tratamento clínico. Acta Fisiatr 2005; 12(2):55-60.

20. Wakefield B, Holman JH. Functional trajectories associated with hospitalization in older adults. Western J N urs Res 2007; 2:161-177.

21. Andrade OG. Cuidado ao idoso com sequela de acidente vascular cerebral: representações do cuidado familiar [dissertação]. Ribeirão Preto (SP): Escola de Enfermagem de Ribeirão Preto, Universidade de São Paulo; 1996.

22. Karsh UM. Envelhecimento com dependência: revelando cuidadores. São Paulo: Educ; 1998.

23. Hager K, Nennmann U. Rehabilitation of the elderly - influence of age, sex, mains diagnoses and activities of daily living ( $A D L$ ) on the elderly patients' return to the previous living conditions. Arch. Gerontol. Geriatr. 1997; 25:131-139.

24. Ramos LR, Simões EJ, Albert MS. Dependence in activities of daily living and cognitive impairment strongly predicted mortality in older urban residents in Brazil: a 2-year follow-up. JAGS 2001; 49:1168-1175.

25. Kramer AM. Rehabilitation care and outcomes from the patient's perspective. M ed Care 1997; 35(6).

26. Brasil. M inistério da Saúde. Portaria no 2.528 de 19 de outubro de 2006. Aprova a Política Nacional de Saúde da Pessoa I dosa. Diário Oficial da U nião 2006; 20 out.

27. Atienza A, Stephens MAP, Townsend AL. Role stressors as predictors of changes in womens'optimistic expectations. Pers Indiv Differ 2004; 37:471-484.

Artigo apresentado em 05/03/2008

Aprovado em 27/04/2009

Versão final apresentada em 20/05/2009 